\title{
Blood homocysteine and folic acid levels may provide reference value for the treatment of sudden total frequency deafness
}

\author{
Yang Huang ${ }^{1,2}$, Tao $\mathrm{Lv}^{3}$, Minqiang Xie ${ }^{1}$, Jing $\mathrm{He}^{3}$, Jiahong Pei ${ }^{2}$, Yanfei Guan ${ }^{2}$, Hequn Jiang ${ }^{2}$, \\ Shunmin $\mathrm{Gong}^{2}$, Xianbao $\mathrm{Cao}^{2}$ \\ ${ }^{1}$ Department of Otorhinolaryngology, Zhujiang Hospital of Southern Medical University, Guangzhou 510280, China; ${ }^{2}$ Department of \\ Otorhinolaryngology, The First People's Hospital of Yunnan Province, The Affiliated Hospital of Kunming University of Science and Technology, \\ Kunming 650032, China; ${ }^{3}$ Department of Genetic Diagnosis Center, The First People’s Hospital of Yunnan Province, Kunming 650032, China \\ Contributions: (I) Conception and design: Y Huang, M Xie; (II) Administrative support: M Xie; (III) Provision of study materials or patients: Y Huang, \\ T Lv, M Xie, J He, J Pei; (IV) Collection and assembly of data: Y Guan, H Jiang, S Gong, X Cao; (V) Data analysis and interpretation: Y Huang; \\ (VI) Manuscript writing: All authors; (VII) Final approval of manuscript: All authors. \\ Correspondence to: Minqiang Xie. Department of Otorhinolaryngology, Zhujiang Hospital of Southern Medical University, Guangzhou 510280, \\ China. Email: min_qiang_x@hotmail.com.
}

\begin{abstract}
Background: The cause of sudden sensorineural hearing loss (SSNHL) is still unknown. Literature has indicated that there is a statistically significant correlation between hyperhomocysteinemia and SSNHL, yet there is lack of study in the relationship concerning total frequency deafness subtype of SSNHL. This study investigated the relationship between plasma concentration of homocysteine (Hcy), serum concentration of folic acid and occurrence and treatment responding in total frequency deafness adult patients, and explored whether targeted early intervention was associated with improved clinical outcome in this subgroup.

Methods: A total of 54 consecutive adult patients with diagnosis of sudden total frequency deafness in a single institution was enrolled into the study group. Two control groups were established. Control group 1 was derived from inpatients with normal listening comprehension. Control group 2 included 52 patients with sudden total frequency deafness treated in a parallel hospital. Blood concentration of folic acid and Hcy was investigated. Treatment included Ginkgo biloba extract, dexamethasone, hyperbaric oxygen, folic acid, vitamin B6, and optional vitamin B12. All data was statistically analyzed. Blood level of Hcy and folic acid was compared between study group and control group 1 .

Results: Although there was no clear evidence for the divergence trend of Hcy and folic acid levels individually, the results showed that the study group had higher blood level of Hcy and lower blood level of folic acid, than control group. In the study group, 24 patients (44.44\%) demonstrated treatment effectiveness after the 2 -week treatment course. Patients without vertigo had higher effective rate than patients with vertigo $(\mathrm{P}<0.05)$.

Conclusions: Effective rate of study group was higher than control group 2 which had no folic acid and vitamin B6/B12 supplement. High blood Hcy and low blood folic acid were closely associated in patients with sudden total frequency deafness. The currently accepted concept of treatment for sudden total frequency deafness is not essentially satisfactory. Testing of plasma Hcy and serum folic acid may provide referential value for its treatment and prognosis evaluation.
\end{abstract}

Keywords: Sudden total frequency deafness; homocysteine (Hcy); folic acid; treatment

Submitted May 16, 2019. Accepted for publication Sep 24, 2019.

doi: $10.21037 /$ apm.2019.10.08

View this article at: http://dx.doi.org/10.21037/apm.2019.10.08 


\section{Introduction}

Sudden sensorineural hearing loss (SSNHL) is defined as a hearing loss of at least $30 \mathrm{~dB}$ in 3 contiguous frequencies occurring within 3 days (1). The incidence of SSNHL is estimated to be approximately 10 cases per 100,000 person-years, but it only reflects patients who sought treatment (2). SSNHL impacts significantly on quality of life, resulting in poorer physical and mental health outcomes (3). The cause of SSNHL is still unknown. The most common pathological mechanisms of SSNHL to date include infection (viral and bacterial), cardiovascular disease, inflammation and immunological factors, genetic mutations, and central nerve system abnormalities (4). Thus, the treatment of SSNHL is controversial. Its recovery potential depends on a number of factors including patient age, presence of vertigo at onset, degree of hearing loss, the time between the onset of hearing loss and treatment, and audiometric configuration (5).

According to the different audiogram profiles and different treatment outcomes, researchers tended to defined SSNHL into several sub-types: low-frequencies descending, high-frequencies descending, flat descending, and total frequency deafness $(6,7)$. Total frequency deafness is described as suddenly deafness occurring within 72 hours, hearing loss at all frequencies with average hearing threshold at 250 to $8,000 \mathrm{~Hz}(250,500,1,000,2,000$, $3,000,4,000,8,000 \mathrm{~Hz}$ ) and profound hearing loss over $80 \mathrm{~dB}$ HL. According to recent reports, low-frequencies descending, the type with better prognosis may be related to the possible cause of endolymphatic hydrops, which is sensitive to medicine. On the contrary, total frequency deafness, with type with poor prognosis may be related to its possible pathogenesis of inner ear embolism or thrombosis $(8,9)$. However, the vascular etiology of SSNHL described in recent literature remains debatable. A systematic review found that smoking, increased alcohol consumption, and inherited prothrombophilic mutations such as factor $\mathrm{V}$ Leiden and MTHFR gene polymorphisms were more frequently observed in patients with SSNHL than in the general population (10). However, diabetes, hypertension, and hyperlipidemia are not associated with the risk of SSNHL in the general population. So far, cardiovascular risk factors have not demonstrate consistent impact pattern.

Due to the significant impact of hearing loss, identifying potentially modifiable risk factors and markers for this condition is a public health priority. Literature has indicated that there is a statistically significant correlation between hyperhomocysteinemia and SSNHL (11-14). Conversely, Cadoni et al. (15). reported there was no such correlation. However, these studies enrolled all types of SSNHL rather than narrowing on the type of total frequency deafness which may be more attributed to vascular factors. Therefore, it is necessary to identify the correlation between blood concentration of homocysteine (Hcy) and total frequency deafness. As an important nutrient factor, folic acid level evidently influences the metabolic pathway of Hcy.

This study investigated the relationship between plasma concentration of Hcy, serum concentration of folic acid and occurrence and response to treatment for total frequency deafness adult patients. It was hypothesized that higher plasma Hcy with lower serum folic acid would be associated with high risk of sudden total frequency deafness. In addition, we explored whether the targeted early intervention was associated with improved clinical outcome in total frequency deafness adult patients.

\section{Methods}

From November 2013 to June 2016, a total of 54 consecutive adult patients with diagnosis of sudden total frequency deafness in a single institution were enrolled as study group. Existence of other brain damage, Meniere's disease, acoustic neuroma, large vestibular aqueduct syndrome, and abrupt hearing loss with a clear cause (such as trauma, medication, and noise exposure) were excluded. SSNHL was diagnosed according to the criteria of the 2015 American Academy of Otolaryngology-Head and Neck Surgery (AAO-HNS, 2015).

Two control groups were established. Control group 1 was derived from inpatients with normal listening comprehension. They were selected randomly from registration of the institution and were stratified according to age and sex. For the purpose of treatment effect comparison, control group 2 included 52 patients with sudden total frequency deafness type from 2012 to 2014 in a parallel hospital (16). Clinical data including demographics, treatment strategy and hearing threshold were analyzed.

Both study group and control group 1 were admitted with related hearing tests, including pure tone valve test, acoustic impedance test, and auditory brainstem response (ABR). Related imaging studies (cranial MRI or internal auditory MRI) for extracranial disease were performed. Result of tympanic examination was normal. Study group patients underwent pure audiometry 


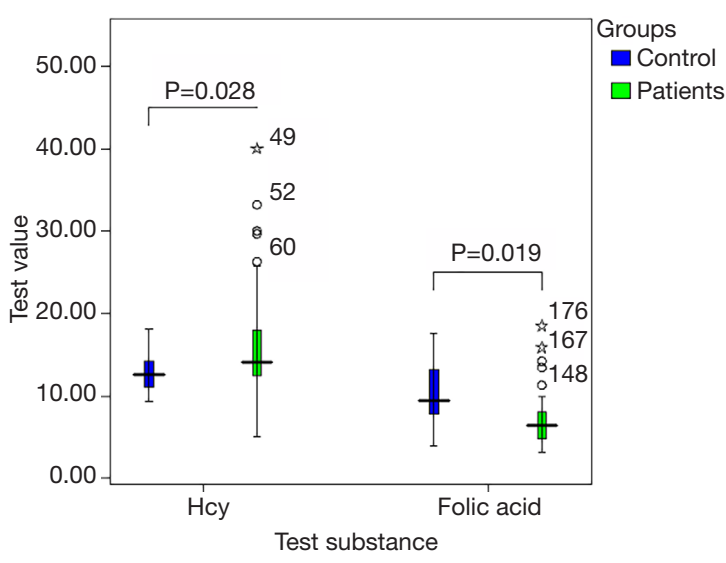

Figure 1 The Boxplots shows the association of Hcy and folic acid levels between the patients and control. Hcy, homocysteine.

examination at 2 weeks after treatment.

We used a unified questionnaire to investigate patients of study group and control group one, including general conditions, diet, lifestyle, disease history, and medication history. After consenting to be enrolled in the study, each patient had $5 \mathrm{~mL}$ of venous blood collected on site. The samples were anticoagulated with EDTA to separate plasma, and placed in a refrigerator at $-20^{\circ} \mathrm{C}$. As red blood cells are where Hcy is primarily produced, they continue to produce Hcy and release into the extracellular fluid during the period of contraction and coagulation ex vivo. Being stored at room temperature for 1 hour after separation, the Hcy concentration in the blood sample can increase by about $10 \%$. Therefore, serum Hcy is higher than plasma. However, if the collected blood sample is immediately put into ice bath or the plasma is separated within 1 hour, an abnormal increase in serum Hcy will be avoided. Blood samples were obtained from all patients after fasting overnight. None of the patients with complete spasticity received sulfasalazine and/or 5-aminosalicylic acid, glucocorticoids, immunosuppressive agents, and folic acid and vitamin B12 within 1 month before blood sampling. Plasma folic acid level was determined by an ELECSYS-170 autoanalyzer using commercial kits (Roche Diagnostics GmbH, Mannheim, Germany). Hcy levels were determined with a Shimadzu HPLC analyzer using Recipe commercial kits (Recipe Chemical and Instruments GmbH, Munich, Germany).

Based on multi-center recommendations (17), the main treatment strategy for study group was intravenous injection of Ginkgo biloba extract and dexamethasone, with hyperbaric oxygen, for a course of 2 weeks: Ginkgo biloba extract $20 \mathrm{~mL} /$ day; dexamethasone $10 \mathrm{mg} /$ day, 5 days then reduced to oral $10 \mathrm{mg}$ for 3 days; then reduced to $5 \mathrm{mg}$ for 2 days before withdrawal. Patients received concomitant hyperbaric oxygen treatment for 10 days. Defibrinogen drugs were not used. Oral Hcy moderation drugs were administered with reference to the regimen for cardiovascular and cerebrovascular diseases: folic acid tablets $0.65 \mathrm{mg}$ /day, plus vitamin B6 and B12, 1 tablet t.i.d.

The hearing test-based evaluation system for treatment outcome after 2 weeks has four grades. To specify, "Healed" means the average hearing threshold recovered to normal; "Significant" means the average hearing threshold at the impaired frequency is improved by $\geq 30 \mathrm{~dB}$; "Effective" means the average hearing threshold at the impaired frequency is improved by $\geq 15 \mathrm{~dB}$; "Ineffective" means the average hearing threshold of impairment frequency is improved by $<15 \mathrm{~dB}$. For the analysis of the treatment results of study group with complete data in this study, we compared and reported the therapeutic effects of 52 cases of sudden total frequency deafness in a hospital from 2012 to 2014 after 2 weeks (16).

All data were analyzed using SPSS19.0 statistical software. All data were expressed as mean and standard deviation. One-way analysis of variance was used to compare the mean value of results of the two groups. $\chi^{2}$ test, rate of multiple comparisons and other methods were used for statistical analysis, with $\mathrm{P}<0.05$ as statistically significant.

\section{Results}

The study group had 54 patients who were diagnosed as total frequency deafness type based on clinical features and audiometry only. The average age was 36.5 [18-69] years. Thirteen patients $(24.07 \%)$ were accompanied with vertigo; 11 patients $(20.37 \%)$ were accompanied with systemic diseases (including diabetes and hypertension). The disease course of every patient was $\leq 2$ weeks. The average age of control group 1 was 34.7 [20-65] years. The demographic data and questionnaire parameters showed no significant difference between the two groups. After blood sample collection, patients in the study group received 2-week treatment before taking pure audiometry test.

Test items and results are demonstrated below (Figure 1). Concentration of Hcy and folic acid before treatment was compared between the study group and control group 1 (Table 1). Although there was no clear evidence for the negative association of Hcy and folic acid levels, the results showed that 
Table 1 Comparison of Hcy and folic acid levels between the patients and control

\begin{tabular}{lcccc}
\hline Parameters & Control $(\mathrm{n}=45)$ & Patients $(\mathrm{n}=54)$ & $\mathrm{t}$ & $\mathrm{P}$ (two-sided test) \\
\hline Hcy & $12.59 \pm 2.77$ & $14.77 \pm 6.45$ & -2.249 & 0.028 \\
Folic acid & $9.24 \pm 3.58$ & $7.40 \pm 4.03$ & 2.380 & 0.019 \\
\hline
\end{tabular}

Two independent sample $t$-tests, $\mathrm{P}<0.05$. Hcy, homocysteine.

Table 2 Comparison of the treatment effects of 54 cases of our study with reporting 52 cases of sudden total frequency deafness in a parallel hospital after 2 weeks treatment

\begin{tabular}{lcccc}
\hline Parameters & Healed, $\mathrm{n}(\%)$ & Significantly, $\mathrm{n}(\%)$ & Effectiveness, $\mathrm{n}(\%)$ & Invalid, $\mathrm{n}(\%)$ \\
\hline Our study $(\mathrm{n}=54)$ & $7(12.96)$ & $7(12.96)$ & $10(18.52)$ & $30(55.56)$ \\
Control 2 $(\mathrm{n}=52)$ & $5(9.62)$ & $12(23.08)$ & $13(25.00)$ & $22(42.31)$ \\
\hline
\end{tabular}

the study group had higher blood Hcy and lower blood folic acid, than control group. Twenty-four patients $(44.44 \%)$ in the study group, after 2 weeks treatment, had positive response to treatment (including healed, significant and effective). Patients without vertigo had higher positive response rate than patients with vertigo $(\mathrm{P}<0.05)$.

Study group patients were compared for the treatment effect against 52 patients in a parallel hospital (16) during the second week. The only difference of treatment strategy between the groups was folic acid and vitamin B6/B12. The sub-grades difference was not statistically significant, although the total effective rate was significantly higher than control group 2 which did not receive folic acid and vitamin B6/B12 supplement (Table 2).

\section{Discussions}

Our study found that the plasma Hcy level was elevated and serum folic acid level decreased in patients with sudden total frequency deafness, with statistical difference compared with the normal control group $(\mathrm{P}<0.05)$. Is Hcy a new risk factor for vascular disease related to SSNHL, or some particular subtypes of SSNHL? There is still contradiction in the evidence of vascular etiology in the SSNHL literature. It has been reported in the literature that there is a statistically significant correlation between hyperhomocysteinemia and SSNHL (11-14). Conversely, Cadoni et al. (15) denied the correlation between them. Berner et al. (17) studied 91 Danish adults (67-88 years old) and found no association between serum folic acid, vitamin B12 and Hcy and hearing loss. Gopinath et al. (18) also reported in a cross-sectional study that serum folic acid, vitamin B12, and Hcy concentrations were not significantly associated with sudden hearing loss; however, folic acid serum concentration and Hcy was associated with senile deafness. Fusconi (19) conducted a meta-analysis of thrombophilia and SSNHL and also pointed out that Hcy appeared to be somewhat inadequate as a risk factor for subcortical small vessel disease (SSVD). Our previous study showed that the level of Hcy and folic acid in un-grouped SSNHL cases were not statistically significant compared with the control group, which was consistent with the result of Cadoni et al. (15). However, with narrowing down to the subgroup, plasma Hcy level in patients with total frequency deafness type was elevated and serum folic acid was decreased, both with statistic difference verse the control group $(\mathrm{P}<0.05)$. Studies showed $(20)$ that plasma fibrinogen and cholesterol levels were significantly higher in SSNHL patients than in the general population. Congenital and acquired thrombophilia may be the cause of SSNHL (21). Therefore, we hypothesize that sudden total frequency deafness may share a common cause with stroke: internal ear thrombosis or thrombosis. Elevated Hcy level may suggest vascular embolism or thrombosis in the inner ear. Hyperhomocysteinemia may be a risk factor for sudden total frequency deafness.

Currently, there are two very important recognized aspects in the formation of hyperhomocysteinemia: (I) nutritional factors, namely, the deficiency of the metabolic co-factors vitamin B6, vitamin B12, and/or folic acid. Since vitamin B12 is a coenzyme of methionine synthetase, folic acid is a donor of methyl in the body. The absence of both can cause disease methylenetetrahydrofolate reductase (MTHFR) and cystathionine- $\beta$-synthase. The decrease 
of cystathionine- $\beta$-synthase (CBS) activity hinders the regeneration of methionine, resulting in the accumulation of Hcy in the body. Therefore, folic acid metabolism is considered to be the most important factor affecting the level of Hcy (22). Serum folate and vitamin B12 levels are negatively correlated with plasma Hcy levels. The lower the levels of folic acid and Vitamin B12, the higher the level of plasma Hcy. Hcy synthesis and metabolic pathways and their associated enzyme system defects, nutritional deficiencies (folate, vitamin B12, and vitamin B6) can all cause hyperhomocysteinemia. (II) Genetic factors, such as MTHFR, CBS gene mutations to reduce enzyme activity, etc., can lead to accumulation of Hcy in the body. The process of Hcy remethylation to methionine S-adenosine transferase (MAT) requires the participation of MTHFR. MTHFR deficiency is an autosomal recessive inheritance disease. The most common mutation in the MTHFR gene is C677T. This mutation results in decreased enzyme activity and increased thermal instability of the enzyme, which in turn leads to elevated plasma Hcy levels. Compared with the mutant heterozygote (CT), homozygous mutant (TT) homozygous mutants results in a significant decrease in enzyme activity and increased Hcy levels. However, if the folic acid in individuals with MTHFR gene C677T homozygous mutation (TT) is above the middle level, hyperhomocysteinemia may not occur. Meanwhile, if folic acid is in deficiency, it will result in mild to severe hyperhomocysteinemia. This phenomenon suggests that genes and nutrients may interact with each other (23). Cystathionine- $\beta$-synthase is an important enzyme in the trans-sulfur process. Among them, T833CG919A mutation can lead to decrease of enzyme activity and increase of Hcy. Rare homozygous mutation of CBS, with clinical manifestation of homocystinuria, may have Hcy level up to $400 \mu \mathrm{mol} / \mathrm{L}$.

Possible mechanisms of Hcy-damaged hearing including: (I) due to the production of over/superoxide, damage of vascular endothelial cells and change of coagulation factor function increase thrombosis forming potential; (II) activation of Hcy forms a dense complex with apolipoprotein $\mathrm{B}$, which is easy to engulfed by macrophages in the blood vessel wall, causing accumulation of fat in the blood vessel wall; (III) Hcy can facilitate arterial embolization of small arteries and promote the proliferation of vascular smooth muscle cells involved in the formation of atherosclerosis; (IV) Hcy can affect the transmethylation reaction in vivo, causing DNA damage, affecting cells Hcy interferes with glutathione synthesis, and glutathione interacts with nitric oxide to protect blood vessels. The normal plasma total Hcy level in fasting plasma is $5-15 \mu \mathrm{mol} / \mathrm{L}$. Ideally it should be $<10$, and $15 \mu \mathrm{mol} / \mathrm{L}$ is considered to be the cut off level of hyperhomocysteinemia. The level of plasma Hcy increases with age, and the level of Hcy in male is significantly higher than that in females, especially in those over 45 years of age. Premenopausal women usually have a $20 \%$ lower Hcy concentration than men but the level will rise to the same as men after menopause.

The currently accepted view for the treatment of sudden total frequency deafness is not essentially satisfactory even if dexamethasone is added early and drugs to improve microcirculation are used. It is recommended that combination therapy be used as soon as possible, including the reduction of fibrinogen drugs (such as batroxobin) (14). The regimen of folic acid to reduce Hcy takes reference to the conventional dose of treating cardiovascular and cerebrovascular disease. Clinically Hcy-reducing regimen may contain folic acid alone or in combination with vitamin B6, and vitamin B12. Ubbink (24) and Klerk (25) reported similar results: on average, folic acid alone at $0.65 \mathrm{mg} / \mathrm{day}$ is associated with a similar reduction in the concentration of Hcy to folic acid combined with vitamin B6 and vitamin B12, respectively $42 \%$ and $50 \%$. However, in some cases, folic acid treatment alone may cause an abnormal increase in Hcy concentration. In our study, 54 patients with sudden total frequency deafness were compared with the treatment effect of 52 patients in a parallel hospital (16) during the second week. The sub-grades difference was not statistically significant, Although the total effective rate was clearly higher than control group 2 which were not treated by folic acid and vitamin B6, B12. Furthermore, we found that neither group of patients used fibrinogen-lowering drugs (such as batroxobin). Is early fibrinogen-lowering treatment able to increase the effective rate? We believe that the cause of sudden total frequency deafness may be disturbance of vascular circulation in the common cochlea or spiral arterial spiral artery. Some patients may present with elevated D-dimer, suggesting that embolism or thrombosis may have already occurred. Early intervention using thrombolytic drugs is required. However, patients are often out of the window for the optimal thrombolysis timing when presenting, as pathological changes have turned irreversible.

Despite the use of folic acid and vitamin B6, B12 did not improve the clinical outcomes in our study, we suggest that keeping a low level of Hcy in daily life can be helpful in the clinical prevention and treatment of sudden total frequency 
deafness. In addition, our data found that the prognosis of the sudden total frequency deafness without vertigo was better than that with the vertigo group. The reason why it causes insensitivity to treatment remains to be further explored.

Our study has limitations. In addition to gender and age, other factors that affect the concentration of Hcy, such as physical activity, drinking and coffee, smoking and other lifestyle factors can affect the plasma total Hcy concentration. The most important factor is smoking. The amount of smoking is positively correlated with the concentration of Hcy; this relationship is also found between coffee intake and Hcy concentration, especially in men, which is not related to filtration of coffee or not. O'Callaghan et al. (26) found that smoking increased risk of vascular disease, as plasma Hcy $>12 \mu \mathrm{mol} / \mathrm{L}$, the risk increased in sex was even more significant, 12 times than normal Hcy and non-smokers. The authors concluded that smokers with elevated levels of plasma Hcy have a significantly increased risk of developing cardiovascular disease. They observed the combined effect of total cholesterol and Hcy and found that patients with the highest Hcy and cholesterol levels had the highest levels of cardiovascular disease compared with those with low levels. The risk increases by 4 times (26). In our study, only study group and control group 1 completed the lifestyle questionnaire, such as diet, vitamin intake, smoking and alcohol consumption, etc. The small number and incomplete data prevented us from further analyzing risk factors and outcomes difference of control group 2. Therefore, the interaction between nutrients and lifestyle were not addressed in this study.

\section{Acknowledgments}

Funding: This study was supported by the "KunHua AoXin science and technology planning project" (2014-2017) and "Genetic Diagnosis Center of laboratory open subject" (2017ZDKFKT003) in the First People's Hospital of Yunnan Province. Data analyzed in this paper were carried out by the Department of Genetic Diagnosis Center of the Hospital. The assistance provided by the institutes and aforementioned individuals is greatly appreciated.

\section{Footnote}

Conflicts of Interest: The authors have no conflicts of interest to declare.
Ethical Statement: The authors are accountable for all aspects of the work in ensuring that questions related to the accuracy or integrity of any part of the work are appropriately investigated and resolved. The study was approved by institutional Ethics Committee of the First People's Hospital of Yunnan Province (No. 2018GJ145).

\section{References}

1. Khater A, El-Anwar MW, Nofal AA, et al. Sudden sensorineural hearing loss: comparative study of different treatment modalities. Int Arch Otorhinolaryngol 2018;22:245-9.

2. Li-Korotky HS. Age-related hearing loss: quality of care for quality of life. Gerontologist 2012;52:265-71.

3. Härkönen K, Kivekäs I, Rautiainen M, et al. Quality of life and hearing eight years after sudden sensorineural hearing loss. Laryngoscope 2017;127:927-31.

4. Chau JK, Lin JR, Atashband S, et al. Systematic review of the evidence for the etiology of adult sudden sensorineural hearing loss. Laryngoscope 2010;120:1011-21.

5. Conlin AE, Parnes LS. Treatment of sudden sensorineural hearing loss: I. a systematic review. Arch Otolaryngol Head Neck Surg 2007;133:573-81.

6. Editorial Board of Chinese Journal of Otorhinolaryngology Head and Neck Surgery, Society of Otorhinolaryngology Head and Neck Surgery, Chinese Medical Association. Guideline of diagnosis and treatment of sudden deafness (2015). Zhonghua Er Bi Yan Hou Tou Jing Wai Ke Za Zhi 2015;50:443-7.

7. Michel O, Deutsche Gesellschaft für Hals-Nasen-OhrenHeilkunde, Kopf- und Hals-Chirurgie. The revised version of the german guidelines "sudden idiopathic sensorineural hearing loss". Laryngorhinootologie 2011;90:290-3.

8. Roh KJ, Lee EJ, Park AY, et al. Long-term outcomes of acute low-tone hearing loss. J Audiol Otol 2015;19:74-8.

9. Stachler RJ, Chandrasekhar SS, Archer SM, et al. Clinical practice guideline: sudden hearing loss. Otolaryngol Head Neck Surg 2012;146:S1-35.

10. Lin RJ, Krall R, Westerberg BD, et al. Systematic review and meta-analysis of the risk factors for sudden sensorineural hearing loss in adults. Laryngoscope 2012;122:624-35.

11. Capaccio P, Ottaviani F, Cuccarini V, et al. Genetic and acquired prothrombotic risk factors and sudden hearing loss. Laryngoscope 2007;117:547-51.

12. Lee EJ, Cho YJ, Yoon YJ. Methylenetetrahydrofolate 
reductase $\mathrm{C677T}$ gene mutation as risk factor for sudden sensorineural hearing loss: association with plasma homocysteine, folate and cholesterol concentrations. J Laryngol Otol 2010;124:1268-73.

13. Marcucci R, Alessandrello Liotta A, Cellai AP, et al. Cardiovascular and thrombophilic risk factors for idiopathic sudden sensorineural hearing loss. J Thromb Haemost 2005;3:929-34.

14. Uchida Y, Sugiura S, Ando F, et al. Association of the C677T polymorphism in the methylenetetrahydrofolate reductase gene with sudden sensorineural hearing loss. Laryngoscope 2010;120:791-5.

15. Cadoni G, Scipione S, Agostino S, et al. Coenzyme Q 10 and cardiovascular risk factors in idiopathic sudden sensorineural hearing loss patients. Otol Neurotol 2007;28:878-83.

16. Zhang HR, Sun YP, Chen H, et al. Analysis on clinical prognosis of sudden total frequency deafness. Chin J Otorhinolaryngol Skull Base Surg 2016;22:54-7.

17. China Multi-Center Clinical Research Collaborative Group of Sudden Deafness. Multi-center clinical study of sudden deafness treatment in China. Chin J Otorhinolaryngol Head Neck Surg 2013;48:355-61.

18. Gopinath B, Flood VM, Rochtchina E, et al. Serum homocysteine and folate concentrations are associated with prevalent age-related hearing loss. J Nutr 2010;140:1469-74.

Cite this article as: Huang Y, Lv T, Xie M, He J, Pei J, Guan Y, Jiang H, Gong S, Cao X. Blood homocysteine and folic acid levels may provide reference value for the treatment of sudden total frequency deafness. Ann Palliat Med 2019;8(5):604-610. doi: 10.21037/apm.2019.10.08
19. Fusconi M, Chistolini A, de Virgilio A, et al. Sudden sensorineural hearing loss: a vascular cause? Analysis of prothrombotic risk factors in head and neck. Int J Audiol 2012;51:800-5.

20. Fusconi M, Chistolini A, Angelosanto N, et al. Role of genetic and acquired prothrombotic risk factors in genesis of sudden sensorineural hearing loss. Audiol Neurootol 2011;16:185-90.

21. Rudack C, Langer C, Stoll W, et al. Vascular risk factors in sudden hearing loss. Thromb Haemost 2006;95:454-61.

22. Capaccio P, Ottaviani F, Cuccarini V, et al. Sudden hearing loss and MTHFR 677C>T/1298A>C gene polymorphisms. Genet Med 2005;7:206-8.

23. Sohn KJ, Croxford R, Yates Z, et al. Effect of the methylenetetrahydrofolate reductase C677T polymorphism on chemosensitivity of colon and breast cancer cells to 5-fluorouracil and methotrexate. J Natl Cancer Inst 2004;96:134-44.

24. Ubbink JB. The role of vitamins in the pathogenesis and treatment of hyperhomocyst(e)inaemia. J Inherit Metab Dis 1997;20:316-25.

25. Klerk M, Verhoef P, Verbruggen B, et al. Effect of homocysteine reduction by B-vitamin supplementation on markers of clotting activation. Thromb Haemost 2002;88:230-5.

26. O'Callaghan P, Meleady R, Fitzgerald T, et al. Smoking and plasma homocysteine. Eur Heart J 2002;23:1580-6. 